\title{
Pristinism under Pressure: Ruetsche on the Interpretation of Quantum Theories
}

\author{
Laura Ruetsche, Interpreting Quantum Theories, \\ Oxford University Press, Oxford, 2011, xvii +379 pp
}

\author{
Simon Friederich
}

Received: 24 October 2011/Accepted: 5 December 2012/Published online: 15 December 2012 (C) The Author(s) 2012. This article is published with open access at Springerlink.com

Interpreting Quantum Theories attempts and achieves no less than three different goals. First, it provides an accessible introduction to the conceptual foundations of the algebraic approach to quantum theories - today's most promising rigorous framework for the formulation of quantum theories that includes those applying to systems with an infinite number of degrees of freedom. Second, it presents the most important interpretive challenges posed by these latter theories, collectively referred to by Ruetsche as " $\mathrm{QM}{ }_{\infty}$ ". Third, it relates the problem of interpreting $\mathrm{QM}_{\infty}$ to more general topics in the philosophy of science, in particular the much discussed issue of scientific realism.

The overall structure of the book is as follows: Chapter 1 sets the stage for what follows by providing some background considerations in terms of more general philosophy of science on what it means to interpret a physical theory. Chapters 2-4 introduce the conceptual basics of the algebraic formulation of quantum theories with particular emphasis on how it generalises standard Hilbert space quantum mechanics ("QM"). After a review of axiomatic approaches in Chapter 5, some apparently very natural, yet competing, interpretive strategies for theories of $\mathrm{QM}_{\infty}$ are outlined in Chapter 6, discussed in some detail here further below. Chapters 7 and 8 focus on one particular challenge posed by the interpretation of $\mathrm{QM}_{\infty}$, namely that in these theories, unlike in ordinary QM, the algebras of linear operators used to describe quantum systems typically do not include any finite-dimensional projections. One of the startling implications of this is that the standard preparation recipes for quantum states as familiar from ordinary QM become problematic in the context of $\mathrm{QM}_{\infty}$ in that no operators projecting the pre-measurement state on a putative "collapsed" or (appealing to Lüders' Rule as a generalised version of the projection postulate) "Lüders conditionalized state"1 are at hand. On similar grounds, the issue

\footnotetext{
${ }^{1}$ See p. 171. Where not specified otherwise, quotations are from "Interpreting Quantum Theories".

S. Friederich $(\bowtie)$

Philosophisches Seminar, Universität Göttingen, Humboldtallee 19, 37073 Göttingen, Germany

e-mail: email@simonfriederich.eu
} 
of the bearers of probabilities seems much more complicated in $\mathrm{QM}_{\infty}$ than in ordinary QM.

The next chapters of the book, Chapters 9-11, turn to the question of whether theories of $\mathrm{QM}_{\infty}$, as formulated in the algebraic framework, can be construed as being about entities that deserve being called "elementary particles". Ruetsche introduces and discusses a number of fascinating effects and phenomena that bear on this issue (namely, the Unruh effect, coherent states, Haag's theorem, and cosmological particle creation), to arrive at the conclusion that all we can hope for is to interpret $\mathrm{QM}_{\infty}$ in terms of a phenomenological particle notion. Such a notion delivers the prospect of grounding particle phenomenology as familiar from accelerators and detectors without at the same time sustaining a particle ontology in terms of particles as fundamental objects everything in the world consists of.

Chapters 12-14 present Ruetsche's main argument, which she calls the "Coalesced Structures Argument", in favour of the central claim of the book that no single interpretive strategy can account for the full explanatory force of $\mathrm{QM}_{\infty}$ in all contexts. Both this claim itself and the structure and key moves of the argument that supports it are discussed in some detail below. To conclude the present overview, Chapter 15 closes the book by connecting the considerations presented in the previous chapters to more general questions in the philosophy of science.

The interpretive issues Ruetsche addresses require a formulation in the vocabulary of the algebraic approach to quantum theories. Some basic notions of this approach are introduced in the next two paragraphs to make the subsequent discussion of some key ideas of the book accessible to the reader who is not familiar with this conceptual framework.

The algebraic formulation of quantum theories can be construed as a generalisation of the standard Hilbert space formalism of ordinary QM. In ordinary QM, the observables of a quantum system are represented by the self-adjoint elements of an algebra $\mathcal{B}(\mathcal{H})$ of bounded linear operators on a Hilbert space $\mathcal{H}$, and the possible states of the system are expressed as density matrices on $\mathcal{H}$. In the algebraic approach one starts in a more abstract way in terms of a $C^{*}$ algebra ${ }^{2}$ and defines the states $\omega$ as linear functionals from $\mathcal{A}$ into the set $\mathbb{C}$ of complex numbers, such that $\omega(I)=1$ and $\omega\left(A^{*} A\right) \geq 1$ for all $A \in \mathcal{A}$. To a first approximation (at least), one may think of the self-adjoint elements of $A \in \mathcal{A}$ as observables of the system and of $\omega(A)$ as the expectation value of $A$ ascribed by the state $\omega$.

A Hilbert space representation $\pi$ maps the $C^{*}$ algebra $\mathcal{A}$ into an algebra $\mathcal{B}(\mathcal{H})$ of bounded linear operators on a Hilbert space $\mathcal{H}$ while preserving all algebraic relations. In ordinary QM, the image $\pi(\mathcal{A})$ of $\mathcal{A}$ under $\pi$ (or, more precisely, its socalled "strong closure" $\left.\pi(\mathcal{A})^{\prime \prime 3}\right)$ is isomorphic to some $\mathcal{B}(\mathcal{H})$. As a consequence, all representations $\pi$ of $\mathcal{A}$ are unitarily equivalent, which means that for any two representations $\left(\pi_{1}, \mathcal{H}_{1}\right)$ and $\left(\pi_{2}, \mathcal{H}_{2}\right)$ there exists a one-to-one norm-preserving

\footnotetext{
${ }^{2}$ An algebra is a vector space with a bilinear vector product defined on it. A $C^{*}$ algebra is an algebra that is isomorphic to a sub-algebra of some algebra $\mathcal{B}(\mathcal{H})$ of bounded linear operators on a Hilbert space $\mathcal{H}$ that is closed with respect to the adjoint operation *.

${ }^{3}$ A sequence $A_{n}$ of operators on $\mathcal{H}$ is said to converge in the "strong topology" iff $\left.\left|\left(A_{n}-A\right)\right| \psi\right\rangle \mid \mapsto 0$ as $n \mapsto \infty$ for each $|\psi\rangle \in \mathcal{H}$. The "strong closure" of $\pi(\mathcal{A})$ includes all elements of $\pi(\mathcal{A})$ itself, together with the limits of all sequences of $\pi(\mathcal{A})$ in this topology.
} 
linear map $U: \mathcal{H}_{1} \mapsto \mathcal{H}_{2}$ such that $U \pi_{1}(A) U^{-1}=\pi_{2}(A)$ for all $A \in \mathcal{A}$. Schrödinger's wave mechanics and Göttingen matrix mechanics can be seen as different Hilbert space representations of the same algebra of observables that is generated by the Heisenberg commutation relations, and they are widely regarded as two formulations of one and the same theory. The choice of representation is a matter of computational convenience rather than physical significance in this case, but outside the scope of ordinary $\mathrm{QM}$, that is, in the realm of $\mathrm{QM}_{\infty}$ where the number of degrees of freedom is infinite and the algebra $\mathcal{A}$ admits unitarily inequivalent Hilbert space representations, things are far less clear.

For the case of $\mathrm{QM}_{\infty}$, Ruetsche considers two (later three) fundamentally different approaches to the interpretation of the concepts just introduced. The first of these, referred to by Ruetsche, following (Arageorgis 1995), as Hilbert Space Conservatism, adamantly sticks to the conventional perspective of identifying the observables of a quantum theory with the self-adjoint elements of a Hilbert space representation $\pi(\mathcal{A})$ (or, more precisely, with the self-adjoint elements of $\pi(\mathcal{A})^{\prime \prime}$, the closure of $\pi(\mathcal{A})$ in the strong operator topology, see Fn. 3) and possible states $\omega$ of the system with density operators $\rho$ on a Hilbert space $\mathcal{H}$ such that $\omega(A)=\operatorname{Tr}(\rho A)$ for all $A \in \pi(\mathcal{A})$. Doing so, the position makes use of the conceptually fundamental GNS representation theorem ("GNS", for Gel'fand, Naimark and Segal), according to which any state $\omega$ on $\mathcal{A}$ defines a unique (up to unitary equivalence) Hilbert space representation $\pi_{\omega}(\mathcal{A})$ such that for some (cyclic) vector $\left|\xi_{\omega}\right\rangle$ on the Hilbert space $\mathcal{H}$ of $\pi_{\omega}(\mathcal{A})$ all expectation values $\omega(A)$ can be written as $\left\langle\xi_{\omega}\left|\pi_{\omega}(A)\right| \xi_{\omega}\right\rangle$ for all $A \in \mathcal{A}$. Unlike in ordinary QM, not all states $\omega$ on $\mathcal{A}$ are in general expressible as density matrices on the Hilbert space of a single state $\omega$ 's GNS representation. Those which can, for some $\omega$, are said to be in $\omega$ 's folium. States $\omega_{1}, \omega_{2}$ giving rise to unitarily inequivalent GNS representations have different folia. If they are pure states, their folia are disjoint, which means that no state expressible as a density matrix on the Hilbert space $\mathcal{H}_{1}$ of the GNS representation of $\omega_{1}$ can be written as density matrix on the Hilbert space $\mathcal{H}_{2}$ of the GNS representation of $\omega_{2}$, and vice versa. The Hilbert Space Conservative regards as physically possible only the states expressible as density matrices $\rho$ in the strong closure of a single state $\omega$ 's GNS representation $\pi_{\omega}(\mathcal{A})$, that is, only states in the folium of the actual state of the system, whatever it is.

The Hilbert Space Conservative's most important opponent, again in the terminology introduced by (Arageorgis 1995), is the "Algebraic Imperialist". Algebraic Imperialism's central claim is that the abstract $C^{*}$-algebra $\mathcal{A}$ itself contains the full physical content of a quantum theory in the sense that its selfadjoint elements correspond directly to the physical magnitudes characterising the system and the states defined on $\mathcal{A}$ are identified with the system's possible states. For the Algebraic Imperialist, Hilbert space representations $\pi(\mathcal{A})$ of the algebra $\mathcal{A}$ are mere computational tools without any independent physical significance. Algebraic Imperialism and Hilbert Space Conservatism disagree on which states are physically possible - only those in the folium of a particular state $\omega$ according to Hilbert Space Conservatism, or all states defined on the algebra $\mathcal{A}$ according to Algebraic Imperialism. Similarly, they disagree on what constitutes an observablethe self-adjoint elements of some $\pi_{\omega}(\mathcal{A})^{\prime \prime}$ according to Hilbert Space Conservatism 
and the self-adjoint elements of $\mathcal{A}$ itself according to Algebraic Imperialism. In addition to these two approaches, Ruetsche introduces a third which she calls "Universalism". Universalism construes the content of a quantum theory in terms of a specific representation, namely, the direct sum of the GNS representations of all states over $\mathcal{A}$. As far as Ruetsche's critique of the three approaches is concerned, the problems and merits of Universalism do not differ much from those of the two other approaches.

Rather than taking sides in the dispute between the three interpretive strategies just outlined, Ruetsche criticises them all for not being able to account for the full explanatory force which theories of $\mathrm{QM}_{\infty}$ recognisably have. The common feature of all three which she targets as the origin of the difficulties they run into is that they specify the physical content of a quantum theory prior to "the messy business of applying the theory in question to individual problems." ${ }^{4}$ By doing so, these interpretations conform to what Ruetsche calls the "ideal of pristine interpretation". As she explains, this ideal is founded on the idea that the content of a theory $T$ be construed as "invariant under changes in $T$ 's applications, [so that] $T$ won't admit different interpretations in different settings." 5 Ruetsche argues at great length that the shortcoming of "pristine" interpretations to account for the full explanatory force of theories of $\mathrm{QM}_{\infty}$ is precisely due to the fact that they follow the ideal of pristine interpretation. In contrast to this ideal, she develops the conception of an "unpristine interpretation", based on the idea that the content of a physical theory $T$ may depend on how $T$ is actually put to use. So, if $T$ is interpreted in an "unpristine" way, this means that what is regarded as physically possible according to $T$ is dependent on what precise applications of $T$ one has in mind. In an unpristine interpretation of a quantum theory the context of application is taken into account to decide what mathematical objects correspond to (which) physical quantities and which states are physically possible.

Ruetsche doesn't mention a further important point of agreement among the "pristine" interpretations she considers, which seems interesting to note: All of them conceive of quantum states as ontic states quantum systems are in in that for each quantum system there is exactly one true quantum state (the one it "is in") which describes it correctly. While this (standard, or "realist") way of construing quantum states is admittedly very natural, it has long been made responsible by interpreters for being the source of the notorious paradoxical aspects of quantum mechanics such as the measurement problem and the apparent tension between quantum theory and relativity. ${ }^{6}$

\footnotetext{
4 See p. 146.

5 See p. 13.

6 The most discussed alternative approaches to the ontic conception of quantum states are epistemic accounts of quantum states, which interpret quantum states as in some way reflecting the state assigning agents' epistemic relations to the systems states are assigned to rather than these systems' objective properties. Quantum Bayesianism, as developed by Fuchs, Caves and Schack, is perhaps the mostdiscussed such account, see (Fuchs 2010) for a recent exposition. Richard Healey's pragmatist approach to quantum theory proposed in (Healey 2012) offers another non-ontic reading of quantum states that avoids the notorious paradoxes along similar lines.
} 
Considering no collapse interpretations of quantum theory, Ruetsche proposes that the "collapsed" state assigned to a quantum system after measurement may be regarded as an "appropriate predictive instrumentality when considering [further] measurements." 7 According to this proposal, quantum states that are assigned after measurement are not agent-independent descriptions of objective properties of the systems they are assigned to but mere instrumental devices to maximise predictive success. Since post-measurement situations belong to the cases where we can reasonably hope to have the most complete knowledge and control of the situation at issue, there seems to be only a very small step from this idea to a fully-fledged non-ontic view of quantum states that construes them as non-descriptive in general. Furthermore, a non-ontic reading of quantum states, which does not acknowledge any such thing as the quantum state a quantum system is in, seems to go very well with the pragmatic spirit of the "unpristine interpretations" Ruetsche favours.

Ruetsche's main argument for unpristine interpretations, the "Coalesced Structures Argument", is centred on the claim that no pristine interpretation can account for what she calls "phase structure"-the coexistence of macroscopically distinct equilibrium phases at certain values of parameters of a quantum theory such as coupling constants or temperature. The most widely known examples of phase structure include the existence of paramagnetic and ferromagnetic phases of iron at $1,033 \mathrm{~K}$ and of liquid and gaseous phases of water at $373 \mathrm{~K}$. As Ruetsche explains in great detail, the standard account of distinctions between different phases requires the number of degrees of freedom of the system at issue to be infinite. For the systems treated in quantum statistical mechanics, which Ruetsche considers first, phase structure appears in the thermodynamic limit, where the system size is taken to infinity while the ratio between particle number $N$ and volume $V$ is kept constant. Distinctions between phases are defined by discontinuities under variations of the parameters in the values of so-called "macroobservables". Macroobservables are characterised by the fact that their value is constant over the whole system in a given phase. An example of a discontinuity signalling a phase transition is the jump in the density of water when turning from the liquid into the gaseous state or the jump in the magnetic susceptibility of iron when turned from the paramagnetic into the ferromagnetic phase. Troubles for Hilbert Space Conservatism start with the observation, derived and discussed at length in the book, that states corresponding to distinct ("pure") phases have different folia, that is, that they cannot be written as density matrices on a single Hilbert space $\mathcal{H}$. Assuming that different phases are physically possible for one and the same physical system therefore means to regard more states as physically possible than can be represented as density matrices on a common Hilbert space. This implies that Hilbert Space Conservatism cannot account for phase structure by not acknowledging enough states as physically possible.

Algebraic Imperialism faces a different challenge when attempting to account for phase structure. While acknowledging all the necessary states corresponding to distinct phases, the position does not interpret as observables the "macroobservables" in terms of which the phases are defined as distinct in the first place. The reason for this is that the defining properties of macroobservables rule out that they

\footnotetext{
${ }^{7}$ See p. 171.
} 
be elements of the algebra $\mathcal{A}$ (as explained in detail in the book), so that macroobservables simply are not observables in the Algebraic Imperialist's sense. Algebraic Imperialism therefore lacks a criterion to distinguish, and to define in the first place, the different phases the existence (and putative coexistence) of which is to be explained. Universalism, as Ruetsche demonstrates, encounters very similar problems. An additional difficulty that concerns both Algebraic Imperialism and Universalism-the " $W^{*}$ argument", as Ruetsche calls it-is that not all of the states which they acknowledge as physically possible have a well-defined time-evolution on their GNS representation. Regarding states admitting no time-evolution as physically possible seems inherently problematic.

An immediate objection to this line of thought is that real physical systems, to which the conceptual apparatus of quantum statistical mechanics is applied in practice, are characterised only by a finite number of degrees of freedom. To describe them in terms of infinitely many degrees of freedom, it may be argued, means making an idealisation on which no substantive interpretive claims should be based. ${ }^{8}$ Ruetsche herself tends towards the alternative view that interpretations on the assumption of the thermodynamic limit are legitimate in that taking this limit is vital to the explanatory success of quantum statistical mechanics. ${ }^{9}$ She acknowledges, however, that this "idealization complaint" ${ }^{10}$ against her interpretive claims must be taken seriously. To support the Coalesced Structures Argument against it, she turns to conventional ("Lagrangean") quantum field theory (QFT), as used with great success, for instance, in contemporary high energy physics to predict and explain the results of accelerator experiments. Since theories of conventional QFT are formulated in terms of a space-time continuum, challenging the assumption that the number of degrees of freedom is infinite seems more difficult in this context. ${ }^{11}$

A large part of Ruetsche's discussion of the Coalesced Structures Argument in conventional QFT is centred around the notion of spontaneous symmetry breaking (SSB), to which, again, she provides an extremely useful introduction. Intuitively, a state exhibits SSB just in case the symmetries of the underlying laws of motion are radically absent from it (see Chapter 13 for a rigorous criterion of SSB). SSB is closely related to phase structure in that the distinction between states of broken and unbroken symmetry commonly lines up with a distinction between different phases. In conventional QFT, SSB is widely believed to play a fundamental role in the

\footnotetext{
${ }^{8}$ See, for instance, (Callender 2001) and Section 9 of (Earman 2004) for considerations on which this objection might be based.

${ }^{9}$ Ruetsche defends this view by drawing attention to the success of renormalisation group theory in accounting for universality and critical phenomena, which success, as she argues, is crucially based on the idealisations of the thermodynamic limit (see p. 339). This claim can be questioned, however, for instance by pointing to the fact that analyses that employ renormalisation group techniques may still have to take into account the finiteness of real systems to have maximum explanatory success. See (Butterfield and Bouatta 2011) for a study of reduction and emergence in phase transitions that emphasises this point.

10 See p. 330.

11 It might be challenged, however, arguing from the fact that the QFTs of the standard model of elementary particle physics are nowadays widely regarded as effective field theories, whose range of validity does not extend to arbitrarily high energies and arbitrarily small distances.
} 
generation of particle masses in form of the "Higgs mechanism", which is often associated with the notion of a spontaneously broken local gauge symmetry. ${ }^{12}$

As Ruetsche sees it, her strategy to defend the Coalesced Structures Argument by appeal to physicists' QFT faces two major obstacles. The first is that, at least at present, we do not have any rigorous formulation of the most successful theories of conventional QFT in the algebraic framework. Therefore, the question of whether physicists' QFT supports her case against pristine interpretations is at least in part a matter of speculation about future physics.

The second obstacle which she recognises is that the alleged explanatory role of $\mathrm{SSB}$, and hence the status of phase structure in conventional QFT, remain unclear inasmuch the status of SSB in the generation of particle masses through the Higgs mechanism continues to be a matter of debate. ${ }^{13}$ Unfortunately, however, Ruetsche's discussion of gauge symmetry breaking and the Higgs mechanism is misleading in several respects. Its most blatant shortcoming is that it identifies a specific choice of gauge (comparable in its significance to the choice of a coordinate system), namely, the unitary gauge, with "the state of broken symmetry suiting the Higgs mechanism." 14 This is misleading, first, in that a gauge is not a "state" at all, in particular not one exhibiting broken symmetry, and, second, in that making the specific choice of the unitary gauge specifically means eliminating the gauge symmetry completely at an "explicit" level, so that SSB cannot occur at all. ${ }^{15}$ Other choices of gauge fixing do not eliminate the gauge symmetry completely and allow that a remnant global subsymmetry of the local gauge symmetry can still be spontaneously broken. However, the relation between these instances of SSB and phase structure is very complicated in that the distinction between broken and unbroken remnant global gauge symmetry is not in general associated with a distinction between different phases. ${ }^{16}$

As far as local gauge symmetries themselves are concerned, it has to be noted that our current frameworks of quantising gauge theories do not support the notion of a spontaneously broken local gauge symmetry at all. This statement can be made rigorous in the context of lattice gauge theory, where it is known as "Elitzur's theorem". ${ }^{17}$ However, regardless of what one actually sees as the role of SSB in the Higgs mechanism or in particle physics more generally, it is uncontroversial that our most successful theories of conventional QFT exhibit a very rich phase structure. ${ }^{18}$

\footnotetext{
12 See, for instance, Chapter 14 of (Halzen and Martin 1984) for a standard account of the Higgs mechanism in terms of this notion. A gauge symmetry is a symmetry where different variable configurations related by symmetry transformations correspond to the same physical situation. See (Earman 2004), Section 6, for an introduction to gauge symmetries aimed at philosophers.

${ }^{13}$ For a challenge to the conventional perspective on SSB in the Higgs mechanism see, for instance, (Earman 2004), Section 9.

14 See p. 331.

15 The main advantage of the unitary gauge is that it is useful in a classical argument giving an idea as to which degrees of freedom are physical and which can be "gauged away".

16 See (Caudy and Greensite 2008) for a state-of-the-art computational study of this issue and (Friederich 2012) for an interpretive discussion aimed at philosophers.

17 See (Elitzur 1975).

18 See, for instance, (Linde 1979) for a classic reference.
} 
This may be taken to ground some optimism for Ruetsche's hope that these theories, once cast in a rigorous formulation using the algebraic framework, might exhibit "a structure sustaining the Coalesced Structures Argument." 19

As Ruetsche argues in the final chapter of the book, the conclusion that in order to account for the full explanatory force of theories of $\mathrm{QM}_{\infty}$ one has to give an unpristine interpretation of them has important ramifications for more general issues in the philosophy of science. Most importantly, she regards it as indicating a problem for the various brands of scientific realism, which all try to account for the empirical success of our best scientific theories by assuming that they are true, which, as she argues, must be construed as true under a single interpretation. Contrary to this doctrine, the Coalesced Structures Argument suggests that the empirical success of theories of $\mathrm{QM}_{\infty}$ can be accounted for only if different interpretations are given for different contexts of application. As Ruetsche sees it, even sophisticated versions of scientific realism, for instance the position defended by Richard Boyd or the nowadays much discussed Structural Realism in both its "epistemic" and "ontic" varieties, are all incompatible with this idea. Given the importance and tremendous success of quantum theories, this challenge to scientific realism deserves serious consideration. Due to the clarity of Ruetsche's formulation of it the stage is well set for the realists to come up with their rejoinders.

Open Access This article is distributed under the terms of the Creative Commons Attribution License which permits any use, distribution, and reproduction in any medium, provided the original author(s) and the source are credited.

\section{References}

Arageorgis, A. (1995). Fields, particles and curvature: Foundations and philosophical aspects of quantum field theory on curved spacetime and the algebraic approach to QFT, Ph.D. dissertation, University of Pittsburgh.

Butterfield, J., \& Bouatta, N. (2011). Emergence and reduction combined in phase transitions, forthcoming in Frontiers of Fundamental Physics (FFP 11) Conference Proceedings, preprint available at http://arxiv.org/abs/1104.1371.

Callender, C. (2001). Taking thermodynamics too seriously. Studies in History and Philosophy of Modern Physics, 32, 539-554.

Caudy, W., \& Greensite, J. (2008). Ambiguity of spontaneously broken gauge symmetry. Physical Review D, 78, 025018.

Earman, J. (2004). Laws, symmetry, and symmetry breaking: Invariance, conservation principles, and objectivity. Philosophy of Science, 71, 1227-1241.

Elitzur, S. (1975). Impossibility of spontaneously breaking local symmetries. Physical Review D, 12, 3978-3982.

Friederich, S. (2012). Gauge symmetry breaking in gauge theories-in search of clarification. European Journal for Philosophy of Science. doi:10.1007/s13194-012-0061-y.

Fuchs, C. A. (2010). QBism, the perimeter of quantum Bayesianism. arXiv:1003/5209 (forthcoming).

Halzen, F., \& Martin, A. D. (1984). Quarks and Leptons: An introductory course in modern particle physics. New York: Wiley.

Healey, R. (2012). Quantum theory: A pragmatist approach. British Journal for the Philosophy of Science. doi:10.1093/bjps/axr054.

Linde A. D. (1979). Phase transitions in gauge theories and cosmology. Reports on Progress in Physics, 42, 389.

${ }^{19}$ See p. 335. 